Revue scientifique sur la conception et l'aménagement de l'espace

$19 \mid 2018$

L'imagerie du paysage

\title{
Présentation de la rubrique Chercheurs en devenir
}

\author{
Sylvie Servain et Roland Vidal
}

\section{OpenEdition}

\section{Journals}

Édition électronique

URL : http://journals.openedition.org/paysage/297

DOI : $10.4000 /$ paysage.297

ISSN : 1969-6124

\section{Éditeur :}

École nationale supérieure du paysage de Versailles-Marseille, Institut national des sciences appliquées Centre Val de Loire - École de la nature et du paysage, École nationale supérieure d'architecture et de paysage de Bordeaux, École nationale supérieure d'architecture et de paysage de Lille, Agrocampus Angers

Référence électronique

Sylvie Servain et Roland Vidal, «Présentation de la rubrique Chercheurs en devenir », Projets de paysage [En ligne], 19 | 2018, mis en ligne le 01 décembre 2018, consulté le 25 septembre 2020. URL http://journals.openedition.org/paysage/297 ; DOI : https://doi.org/10.4000/paysage.297

Ce document a été généré automatiquement le 25 septembre 2020

Projets de paysage 


\title{
Présentation de la rubrique Chercheurs en devenir
}

\author{
Sylvie Servain et Roland Vidal
}

1 Organisées à l'École nationale supérieure de paysage de Versailles, sous l'égide du ministère de la Transition écologique et solidaire, les septièmes Journées doctorales en paysage ont réuni plus de 70 participants les 3 et 4 avril 2018.

225 communications et 11 posters ont ainsi contribué à faire avancer les débats interdisciplinaires autour de la question du paysage. Les doctorants issus du monde de l'architecture, de l'urbanisme, des sciences de l'environnement, de la géographie et, bien sûr, du paysage ont ainsi pu échanger sur leurs questionnements de recherche et sur les démarches méthodologiques qu'ils mettent en œuvre.

3 Les communications furent réparties en sept sessions traitant successivement des pratiques paysagistes, des politiques publiques, du patrimoine, des processus participatifs, de l'environnement, du paysage urbain et des forêts. Pour autant, au sein de chacune de ces thématiques, les approches ont été particulièrement diversifiées, tant du point de vue de l'échelle traitée - du petit jardin au grand paysage - que du point de vue des disciplines convoquées - des sciences exactes aux pratiques artistiques.

4 Issus d'une sélection rigoureuse ${ }^{1}$, les cinq articles publiés ici ne donnent évidemment pas une vue exhaustive de cette diversité, mais ils en illustrent certains des aspects les plus importants.

5 Tandis qu'Aurélien Ramos aborde la question de l'usage de l'espace public à l'échelle du jardinage urbain, Aurore Meyfroidt analyse un vaste projet de recherche, LOS_DAMA!', portant sur sept régions européennes. Transgressant les échelles, Morgane Robert se penche sur un petit arbuste, le mosa ${ }^{3}$, pour étudier l'impact de cette plante envahissante sur l'ensemble du paysage des Bas de l'île de la Réunion. À cette diversité des échelles s'ajoute celle des champs disciplinaires. En s'intéressant à la perception du paysage par la marche, Ekaterina Shamova, par exemple, se réfère aussi bien à l'ethnoanthropologie qu'à la psychologie, aux neurosciences ou aux disciplines artistiques. Plus proche du paysage tel que défini par la Convention de Florence, Mathilde Rue se 
penche, quant à elle, et à travers la question de l'agroforesterie, sur le lien entre ceux qui créent et entretiennent les paysages de nos campagnes et la manière dont ces paysages sont perçus.

6 Même si elle est loin d'en recouvrir toute l'hétérogénéité, la diversité de ces cinq articles donne une image assez juste de ce qu'est la recherche en paysage.

7 L'objectif n'étant pas de fondre cette diversité en une discipline unique mais d'en accepter la polysémie et d'apprendre aux différents chercheurs à communiquer entre eux, on peut considérer que ces septièmes Journées doctorales ont bien rempli leur rôle.

8 Le programme complet des $7^{\mathrm{e}}$ Journées doctorales en paysage, ainsi que l'ensemble des résumés et des posters sont consultables sur Topia.fr.

\section{NOTES}

1. Tous les communicants ont été invités à proposer un article, 13 d'entre eux ont répondu à cette invitation. Leurs articles ont été soumis au comité scientifique des Journées doctorales qui en a proposé une sélection à la revue Projets de paysage. Cinq d'entre eux ont finalement été retenus et sont publiés ici.

2. LOS_DAMA! (http://www.alpine-space.eu/projects/los_dama/en/home) : Landscape and Open Space Development in Alpine Metropolitan Areas.

3. Nom donné localement à Leucaena leucocephala, également appelé "cassia ", une fabacée proche des acacias et originaire d'Amérique centrale.

\section{AUTEURS}

\section{SYLVIE SERVAIN}

Sylvie Servain est géographe, professeure des universités, département École de la nature et du paysage, Insa Centre Val de Loire, UMR 7324 CITERES (CNRS-université de Tours).

sylvie.servain[at]insa-cvl[dot]fr

\section{ROLAND VIDAL}

Roland Vidal est docteur de l'ENGREF en sciences de l'environnement et ingénieur de recherches à l'École nationale supérieure du paysage de Versailles. Il y codirige le master « Théories et démarches du projet de paysage » et enseigne également à AgroParisTech. Ses activités d'enseignement et de recherche portent sur les territoires d'interface entre la ville, l'agriculture et les espaces naturels.

http://www.rolandvidal.fr/ 